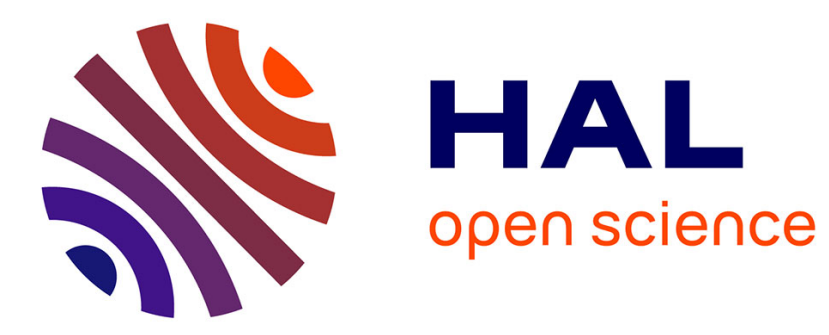

\title{
Ergodicity of the recent geomagnetic field
}

\author{
A. de Santis, E. Qamili, G. Cianchini
}

\section{To cite this version:}

A. de Santis, E. Qamili, G. Cianchini. Ergodicity of the recent geomagnetic field. Physics of the Earth and Planetary Interiors, 2011, 10.1016/j.pepi.2011.04.008 . hal-00759561

\section{HAL Id: hal-00759561 \\ https://hal.science/hal-00759561}

Submitted on 1 Dec 2012

HAL is a multi-disciplinary open access archive for the deposit and dissemination of scientific research documents, whether they are published or not. The documents may come from teaching and research institutions in France or abroad, or from public or private research centers.
L'archive ouverte pluridisciplinaire HAL, est destinée au dépôt et à la diffusion de documents scientifiques de niveau recherche, publiés ou non, émanant des établissements d'enseignement et de recherche français ou étrangers, des laboratoires publics ou privés. 


\section{Accepted Manuscript}

Ergodicity of the recent geomagnetic field

A. De Santis, E. Qamili, G. Cianchini

PII:

DOI:

Reference:

To appear in:

Received Date:

Revised Date:

Accepted Date:
S0031-9201(11)00071-9

10.1016/j.pepi.2011.04.008

PEPI 5403

Physics of the Earth and Planetary Interiors

1 September 2010

11 April 2011

20 April 2011
PHYSICS

OF THE EARTII

AND PLANETARY

INTERIORS

Please cite this article as: De Santis, A., Qamili, E., Cianchini, G., Ergodicity of the recent geomagnetic field, Physics of the Earth and Planetary Interiors (2011), doi: 10.1016/j.pepi.2011.04.008

This is a PDF file of an unedited manuscript that has been accepted for publication. As a service to our customers we are providing this early version of the manuscript. The manuscript will undergo copyediting, typesetting, and review of the resulting proof before it is published in its final form. Please note that during the production process errors may be discovered which could affect the content, and all legal disclaimers that apply to the journal pertain. 


\section{Ergodicity of the recent geomagnetic field}

A. De Santis ${ }^{\mathrm{a}, \mathrm{b},{ }^{*}}$, E. Qamili ${ }^{\mathrm{a}, \mathrm{c}}$ and G. Cianchini ${ }^{\mathrm{a}, \mathrm{c}}$

${ }^{\text {a } I s t i t u t o ~ N a z i o n a l e ~ d i ~ G e o f i s i c a ~ e ~ V u l c a n o l o g i a, ~ S e z i o n e ~ R o m a ~ 2, ~ V i a ~ d i ~ V i g n a ~ M u r a t a ~ 605, ~} 00143$

Roma, Italy.E-mail: angelo.desantis@ingv.it

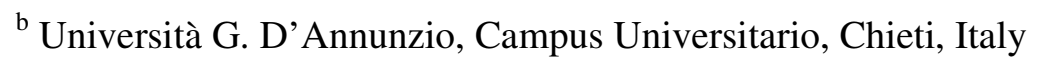

${ }^{\mathrm{c}}$ Scuola di Dottorato in Scienze Polari, Università degli Studi di Siena, Siena, Italy

Rev. April 2011 


\section{Abstract}

The geomagnetic field is a fundamental property of our planet: its study would allow us to understand those processes of Earth's interior, which act in its outer core and produce the main field. Knowledge of whether the field is ergodic, i.e., whether time averages correspond to phase space averages, is an important question since, if this were true, it would point out a strong spatiotemporal coupling among the components of the dynamical system behind the present geomagnetic field generation. Another consequence would be that many computations, usually undertaken with many difficulties in the phase space, can be made in the conventional time domain. We analyse the temporal behaviour of the deviation between predictive and definitive geomagnetic global models for successive intervals from 1965 to 2010, finding a similar exponential growth with time. Also going back in time (at around 1600 and 1900 by using the GUFM1 model) confirms the same findings. This result corroborates previous chaotic analyses made in a reconstructed phase space from geomagnetic observatory time series, confirming the chaotic character of the recent geomagnetic field with no reliable prediction after around 6 years from definitive values, and disclosing the potentiality of estimating important entropic quantities of the field by time averages. Although more tests will be necessary, some of our analyses confirm the efforts to improve the representation of the geomagnetic field with more detailed secular variation and acceleration.

Key words: Geomagnetic Field; Ergodicity; Chaos; Geomagnetic field prediction 


\section{Introduction}

The geomagnetic field is an important property of our planet: its study allows us to understand some processes in Earth's interior, especially in the outer fluid core, where the main field (MF) is produced by turbulent motions of a liquid iron alloy conductive material. A conventional way to study the geomagnetic field at a certain epoch is the Spherical Harmonic Analysis (SHA) that consists of expanding the geomagnetic field potential outside the planet in spherical harmonics as solutions of the Laplace's equation. The result of the analysis is expressed with a set of Gauss coefficients $g_{n}^{m}$ and $h_{n}^{m}$ that completely characterise the corresponding model at each epoch. Then, one can look at the behaviour in time of $g_{n}^{m}$ and $h_{n}^{m}$ to follow the geomagnetic field dynamics. As pointed out by De Santis and Qamili (2010), this conventional analysis, although powerful, has the main drawback that most of the static and dynamical features are obscured by the large contribution of the geomagnetic dipole. An alternative analysis that has been introduced in the recent years is the nonlinear chaotic analysis (NCA): this analysis is expressed mainly in terms of a nonlinear forecasting approach (e.g. Barraclough and De Santis, 1997; De Santis et al., 2002) and is based on the study of the exponential divergence of some prediction from the real signal in the phase space. Indeed, the cornerstone of this analysis is the reconstruction of a pseudo-phase space, taking advantage of a mathematical theorem (Takens, 1981) that establishes a direct way to do it from the time delays of the experimental signal (e.g. Kantz and Schreiber, 1997). A way to connect SHA and NCA has been the introduction of another kind of NCA, directly performed in the usual time domain: this analysis is based on the configurational Shannon Entropy (or its negative quantity, the Shannon Information or Information Content). This quantity can be simply expressed by the Gauss coefficients of the SHA (De Santis et al., 2004; De Santis, 2007, 2008), and its (negative) time derivative is, for chaotic signals, the Kolmogorov entropy $K$ (Wales, 1991). The latter aspect of NCA, to be rigorous, needs the signal under study to satisfy some important properties, the most important of which is its ergodicity, i.e. when time averages are equivalent to phase space averages (e.g. Eckmann and Ruelle, 1985). Therefore, it is an important issue to know whether the field is 
ergodic or not, since if this were true, many computations, usually undertaken with many difficulties in the phase space (e.g. Hongre et al., 1999), would be more easily made in the conventional time domain (De Santis and Qamili, 2010). In addition, ergodicity would be a further evidence for the strong spatio-temporal coupling among the contributions composing the dynamical system that produces and sustains the geomagnetic field (Hongre et al., 1999; De Santis et al., 2003). The strong coupling is typical of many spatio-temporal chaotic phenomena (e.g. Cross and Hohenberg, 1994; Egolf, 2000).

This paper deals with the verification of geomagnetic field ergodicity. In particular it will show that the same kind of chaos, already found in the phase-space reconstruction from several geomagnetic observatory time series (Barraclough and De Santis, 1997; De Santis et al., 2002), also appears in the conventional time domain in terms of an exponential divergence with time between predictive and definitive global geomagnetic field models, thus finding the same results. This outcome will be considered an empirical evidence for the ergodicity of the recent geomagnetic field, so disclosing the potentiality of estimating important entropic quantities by means of time averages.

Ergodicity is important not only in geomagnetism but also in other fields. For instance, in geomorphology the ergodic assumption allows to perform spatial analyses as if they were in time, looking at the temporal cumulated effects on the space, and so without waiting millennia or more to perform the analyses (e.g. Paine, 1985). In engineering seismology, the use of empirical ground motion attenuation relations for site-specific seismic hazard assessment implicitly appeals to the ergodic assumption (e.g. Woo et al., 2006), although there are some techniques that try to overcome this limitation (e.g. Anderson and Brune, 1999; Chen and Tsai, 2002). Ergodicity is also fundamental for studies of some seismic sequences in the time domain where they appear as a chaotic point-process (e.g. De Santis et al., 2010) so that the statistical time analyses can be considered as valid as in the phase space. For many types of laboratory experiments (e.g. lowintensity interference; Buonomano and Bartmann, 1986) assuming the ergodicity from some theoretical ground simplifies most of the computations. Therefore, even though our analysis is 
restricted to the geomagnetic field, the methodology, in principle, can be also extended to other disciplines.

This paper is organized as follows: next section is dedicated to the NCA in the phase space; then we define ergodicity more in detail. The successive section describes the temporal behaviour of the errors between several predicted and definitive global geomagnetic models and how the results are connected with the important question of the ergodicity of the geomagnetic field. Finally, we discuss the results in terms of their significance.

\section{Theory}

\subsection{Nonlinear chaotic analysis of the geomagnetic field in the phase space}

The concept of phase space is a powerful tool for characterizing the dynamical system under study. Let us think of a physical system whose states can be represented by a series of sequential points in a finite-dimension space $\Re^{m}$, i.e. the phase space whose coordinates are all those variables that, at any moment, describe completely the state of the system. During its time evolution, the transition $T_{t_{1} \rightarrow t_{2}}$ from a state $\vec{x}\left(t_{1}\right)$ at time $t_{1}$ to state $\vec{x}\left(t_{2}\right)$ at time $t_{2}$ can be expressed by the equation of motion:

$$
\dot{\vec{x}}(t)=F(\vec{x}(t)), \mathrm{t} \in \mathbb{R}
$$

named flow for the continuous time, or by

$$
x_{n+1}=f\left(x_{n}\right), \mathrm{n} \in \mathbb{Z}
$$

the so-called map for discrete case, where $\vec{x}(t), x_{n} \in \mathfrak{R}^{m}$. A dynamical system is realized by the above equations.

The study of a dynamical system implies the description of the dynamics of the points $\vec{x}(t)$ in the phase space. So, for instance, considering the solution of the discrete case (2) with the initial (condition) state $x_{0}$, the sequence of points $x_{n}=f^{n}\left(x_{0}\right)$ in the phase space is called a trajectory. 
Trajectories can run to infinity or stay confined in a finite volume as time goes on: regularity in their behaviour depends on the form of $F$ (or $f$ for discrete time) and on the chosen initial conditions.

Some studies have shown that the recent geomagnetic field is chaotic (Barraclough and De Santis, 1997; De Santis et al., 2002). This means that any small change $\varepsilon$ of the initial orbit in the phase space propagates exponentially with time, i.e. $\varepsilon(\mathrm{t}) \approx \varepsilon_{0} \mathrm{e}^{K \mathrm{t}}$ where $K$ is the Kolmogorov Entropy (with $K>0$ ), characterising the degree of chaos of the phenomenon under study (e.g. Schuster, 1992). This exponential divergence of close orbits must not be taken in strict sense, but in statistical terms and for short times (theoretically $\mathrm{t} \rightarrow 0$ ). Then we can introduce the mean time $<T>$ after which no reliable prediction can be performed, such as $\langle T\rangle=1 / K$. According to the time delays theorem (Takens, 1981) we do not have to measure all the phase space variables of the dynamical system. Instead, we can reconstruct an equivalent phase space using appropriate successive time delays for each axis, i.e. the so-called embedding space or pseudo-phase space. This procedure allows a onedimensional time series to be converted to a $m$-dimensional representation in order to reveal potential properties of stability or instability of the signal under consideration, such as noise, periodicity or chaos. When the time series is rather short (say, composed of less than 1000 data points) we can apply a nonlinear forecasting approach that consists of subdividing the time series in two equal parts and using the first half to predict the second one in the phase space: no matter the used technique, the way our prediction deviates from the real observation establishes the possible chaoticity of the process related to the studied signal (e.g. Sugihara and May, 1990), and can even allow to quantify it. According to Wales (1991), there is a relation between the Kolmogorov Entropy $K$ and the correlation coefficient $r$ between prediction and actual signal in the form:

$$
\ln (1-r)=q+\alpha K T_{\mathrm{p}}
$$

where $T_{\mathrm{p}}$ is the prediction time in the future, $q$ and $\alpha$ are two empirical constant parameters, with $\alpha=1$ for 1 -D series, $\alpha=2$ for very short multi-dimensional series. De Santis et al. (2002) applied this 
technique to the geomagnetic field secular variation (SV) observed over the past 100 years in 14 observatories non-uniformly distributed over the Earth's surface. They found that the East component of geomagnetic field SV (i.e. the least contaminated by external fields) behaves as a chaotic phenomenon with $\langle T\rangle=1 / K \approx 6$ years, in the sense that no reliable predictions of future geomagnetic field can be made after around 6 years (Barraclough and De Santis, 1997; De Santis et al., 2002).

\subsection{Ergodicity and invariant measures}

A dynamical system is dissipative when, after a transient behaviour, the volume of the phase space containing the initial conditions is contracted by the dynamics, in the sense that as $t \rightarrow \infty$ the points asymptotically visited by the system will be concentrated on a sub-set of $\mathfrak{R}^{m}$ of null Lebesgue measure named attractor (Eckmann and Ruelle, 1985). Since the system does not visit the points on the attractor with the same frequency, a corresponding measure must be introduced. We define the measure $\rho(\vec{x}) d \vec{x}$ as the average time a typical trajectory spends in the elementary volume $d \vec{x}$ of the phase space. Since it can have several solutions strongly depending on the initial conditions, in order to characterize a dynamical system it is necessary to make use of quantities that do not derive from particular realizations of the dynamics. For this purpose, we can observe that there exist subsets $U \supset \Re^{m}$ that are invariant, meaning that they are mapped onto themselves by $f$ (or $F$ )

$$
f(x) \in U, \forall x \in U
$$

when every point in the invariant subset is mapped forward in time by the dynamics (Kantz and Schreiber, 1997). This way the system is characterized by the long term behaviour of its orbit on one of the many invariant subsets, and not by all orbits possibly emerging from all its initial conditions.

Another element of universality comes from the existence of at least one invariant measure inside each set. An invariant measure is such that 


$$
\rho\left(f^{-1}(U)\right)=\rho(U), t>0
$$

where $U \supset \Re^{m}$ and $f^{-1}(U)$ is the set obtained by evolving back in time each point $x \in U$ (Eckmann and Ruelle, 1985). The above equation means that if we have a huge number of points on the invariant subset distributed according to this invariant measure and we apply the time evolution operator $f$ on them, the measure will be exactly reproduced (Kantz and Schreiber, 1997).

All above definitions and concepts are used in order to study dynamical systems and they are part of a general theory named Ergodic Theory (e.g. Asonov, 2001; Walters, 1982).

In general, in this framework, each invariant measure $\rho$ is decomposable into several different pieces. When the measure $\rho$ is no longer decomposable, it is called ergodic. According to the ergodic theorem, for every continuous function $\varphi$ we have:

$$
\lim _{T \rightarrow \infty} \frac{1}{T} \int_{0}^{T} \varphi\left[f^{t}\left(x_{0}\right)\right] d t=\int \rho(d x) \varphi(x)
$$

i.e. a dynamics is called ergodic for almost all initial conditions $x_{0}$ if a time average along a semiinfinite trajectory is identical to space average (Eckmann and Ruelle, 1985); the space average is weighted by the invariant measure $\rho$.

An equivalent definition of ergodicity is that property of a dynamical process that allows the related system to reach any state starting from any other, in the course of its evolution over time; or, in other words, "a system is ergodic if the infinite-time of an observable is independent of the initial condition (except for a set of initial states of measure zero)" (Egolf, 2000).

An important requirement to apply the above concepts is the identification of the invariant set, i.e. the volume of the phase space that remains constant after time evolution: an irreducible attractor, i.e. one composed by one invariant subset only, is what satisfies our request. But in that case the dynamical system has to be dissipative.

We have defined the measure $\rho(\vec{x}) d \vec{x}$ as the average time a typical trajectory spends in the elementary volume $d \vec{x}$ of the phase space. If this measure is independent of initial conditions then 
ergodicity is certain and so space averages computed with respect to $\rho(\vec{x}) d \vec{x}$ equals time averages taken over a typical trajectory. Relationship (6) is the so called Birkhoff's "ergodic theorem" (Walters, 1982): statistically speaking, the system that evolves for a long time "forgets" its initial state.

In the case of the geomagnetic field, the invariant measures are the $K$-entropy and its reversal $<T>=1 / K$, i.e. the limiting mean time of prediction. An outstanding role in ergodic theory and its applications to stochastic processes is played by the various notions of entropy for dynamical systems. If we find that the geomagnetic field is ergodic, this result, in turn, will allow us to simplify most operations related to estimations of its entropy.

\section{Data analyses and results}

Global models of the geomagnetic field are, at the same time, important products and sources of study of the recent and past geomagnetic field. Most of them are made performing a SHA on different magnetic field data, providing sets of Gauss coefficients $g_{n}^{m}$ and $h_{n}^{m}$ at successive epochs or some temporal functions of them. The models may also provide a set of predictive coefficients to estimate close future values of the field.

In the following we will use a technique based on the comparison between predicted and definitive values of the field in a certain period of time. To estimate these values, we will use some different global models, that we will briefly describe below.

The IGRF model (International Geomagnetic Reference Field; Maus et al., 2005; Finlay et al., 2010; internet link: http://www.ngdc.noaa.gov/IAGA/vmod/igrf.html) is a series of mathematical models of the Earth's MF and its SV in terms of a spherical harmonic expansion of the geomagnetic potential. This model comes from a refined combination of some different candidates that are based on diverse criteria of selection among observatory and satellite data from 1900 to 2010 . The core field is represented by a set of Gauss coefficients given at 5-year intervals, with a degree $n$ (and order $m$ ) up to a maximum index $N=10$ (from 1965 to 1995) and 13 (from 2000) and SV up to 
degree $N=8$. In our analyses we use the $10^{\text {th }}$ generation of IGRF (Maus et al., 2005), although a more recent version (the $11^{\text {th }}$ generation) already exists (Finlay et al., 2010).

WMM (World Magnetic Model; Mclean et al., 2004; internet link: http://www.ngdc.noaa.gov/geomag/WMM/DoDWMM.shtml) is an analogous large spatial-scale $\mathrm{SH}$ representation of the Earth's magnetic field. It consists of a degree and order 12 spherical harmonic expansion of the potential of the geomagnetic MF. The model has also an equal number of spherical harmonic SV coefficients predicting the temporal evolution of the field over the upcoming 5-year epoch.

CM4 (Comprehensive Model; Sabaka et al., 2004; http://core2.gsfc.nasa.gov/CM/) is based on observatory hourly means measurements close to 01:00 LT on the quietest day of each month for the period 1960-2000 and every 2 hours for the quietest days during which POGO, MAGSAT, CHAMP and Ørsted satellites flew. The latter satellite data, properly selected, are then included in the model. The internal (core and lithospheric) fields are represented by a degree and order up to $N$ $=65$ internal spherical harmonic expansion. The temporal changes of the coefficients up to degree $N=13$ are described by cubic B-splines (Sabaka et al., 2004). The model takes also into account external ionospheric and magnetospheric fields which are not considered in the present work.

POMME models, versions 3.0 (Maus et al., 2006) and 6.0 (Maus et al., 2010; http://www.geomag.us/models/pomme6.html), are MF models based on CHAMP satellite magnetic measurements (Ørsted and SAC-C satellite data were used only to verify the accuracy of the model). The corresponding input data span from 2000.6 to 2005.7 , centered at 2003.0, for the version 3.0 and from 2000.6 to 2009.7 , centered at 2005 , for the version 6.0. These models reach a maximum degree $N=60$ and include an instantaneous SV and a second time derivative, the so called secular acceleration (SA), both to degree 16.

CHAOS spherical harmonic models, first version (here called CHAOS-1; Olsen et al., 2006) and second version (CHAOS-2; Olsen et al., 2009), are based on high-precision geomagnetic measurements from Ørsted, CHAMP and SAC-C satellites. CHAOS-1 is based on data taken 
between March 1999 and December 2005 centered at 2002.5 whilst CHAOS-2 is an update to 2009.5. CHAOS-1 reaches a maximum degree $N=50$ and includes an instantaneous SV up to degree $N=18$ and a SA up to degree 14 ; CHAOS-2 reaches a maximum degree $N=60$ and includes an instantaneous SV up to degree $N=20$ without including a SA. In order to consider the MF only, in this paper we limit both CHAOS-1 and CHAOS-2 expansions up to $N=14$.

What is common in all above models is that any extrapolation of their calculated geomagnetic field outside the typical time of validity would cause very large errors. How these errors behave in time is important to understand the intrinsic dynamics of the geomagnetic field and its main source, i.e. the outer terrestrial core. We can estimate the errors $\varepsilon$ from predictive and definitive model Gauss coefficients (Maus et al., 2008):

$$
\mathcal{E}=\sqrt{\sum_{n=1}^{N}(n+1) \sum_{m=0}^{n}\left[\left(c_{n}^{m}\right)_{\text {predictive }}-\left(c_{n}^{m}\right)_{\text {definitive }}\right]^{2}}
$$

where $\left(c_{n}^{m}\right)^{2}=\left(g_{n}^{m}\right)^{2}+\left(h_{n}^{m}\right)^{2}$.

Figure 1 shows the errors as estimated from equation (7) for eight temporal segments numbered in progressive order, as taken from the differences between predicted and definitive global geomagnetic models in the period 1965-2010. In this figure we have built the same Figure 1 of Maus et al. (2008) just by digitising all curves. The considered predictive models were WMM for 1975-2010 and IGRF for 1965-2010. As definitive models we considered the CM4 for 1965-2000 and the POMME-3.0 model (Maus et al., 2006) for 2000-2010, with maximum degree 13 and 16, respectively.

Figs. $2 \mathrm{a}$ and $2 \mathrm{~b}$ redraw the same errors for IGRF and WMM, respectively, labelled according to the numbers of Fig.1, but imposing for convenience the same initial time for a better visual inspection and comparison. It is straightforward to see that if we impose the same initial value for both predicted and definitive models, each exponential growth has an offset equal to $-\varepsilon_{0}$ that must be taken into account. Thus, all segments can be represented by an exponential function $s(\mathrm{t})$ such as

$$
s(\mathrm{t})=\varepsilon_{0} e^{\mathrm{t} / \tau}-\varepsilon_{0}=\varepsilon_{0}\left(e^{\mathrm{t} / \tau}-1\right)
$$


where $\varepsilon_{0}$ is an appropriate constant and $\tau$ is the characteristic time of growth: the former is a measure of the initial difference between prediction and actual value, while the latter, in case of an ergodic and chaotic system, is related to K-entropy. As the equation of close but diverging orbits in the phase space, also eq. (8) must be taken in its statistical meaning so that we can write $<\tau>\approx 1 / \mathrm{K}$, where $\langle\tau\rangle$ is the mean value of the characteristic time over many realisations of (8).

For each best-fit curve we indicate the corresponding value of $\tau$ with the associated statistical error (standard deviation). Please note that this error estimation is usually over-optimistic, being internally evaluated among all points of each diverging curve. To have more realistic estimates we calculate mean value and associated standard deviation from all best-fit $\tau$ values for each coherent analysis. In particular, for IGRF $\tau$ has a mean value $<\tau>=5.2$ years (median 5.4 years) with uncertainty ( \pm standard deviation) of \pm 2.3 years (Fig.2a), while for WMM we obtain $\langle\tau>=7.9 \pm 3.7$ years (median 7.6 years; Fig.2b). The larger uncertainty of WMM could be due to some less accuracy of this model with respect to IGRF or, at least, to a more variable accuracy over the time of validity.

If the system is chaotic we will expect an exponential behaviour also for "retrodictions", i.e. when, using the SV at a certain time, the predictive model is forecasted backward in time. To verify this, we reproduced part of the Figure 8 of Maus et al. (2008) where we considered the misfit between the POMME 3.0 (including its SA) and CM4 models, when the former was extrapolated backward from 2002 to 1990. Once referred the time with respect to the epoch 2002 (Fig.3), this segment can be represented by an exponential growth in the reverse time $(t \rightarrow-t)$, i.e. by an exponential decaying function $s^{\prime}(\mathrm{t})$ in the natural time, such as $s^{\prime}(\mathrm{t})=s(-\mathrm{t})=\varepsilon_{0}\left(e^{-\mathrm{t} / \tau}-1\right)$, with $\tau=5.4 \pm 0.5$ years. Also here $\tau$ is the characteristic time associated to the chaotic character of the field.

At this point, a possible criticism could be that the compared (predictive and definitive) models are quite diverse and that their exponential divergence could be just due to the difference between IGRF or WMM model and CM4: this difference becomes obvious in Fig.1, where the estimated 
model errors for the initial times vary significantly. To avoid the problem of the evident model heterogeneity we finally resorted to two couples of more homogeneous models, in terms of model construction and data selection. In particular, we analysed the differences between POMME 3.0 and POMME 6.0 (Maus et al., 2006; 2010) and between CHAOS-1 and CHAOS-2 from 2006 to 2010 (Olsen et al., 2006; 2009), where the older versions of each couple of models acts as a predictive model and the most recent one as the definitive one; POMME models and CHAOS-1 model include their MF, SV and SA terms, so we considered, together with MF and SV terms, all SA terms (case $a$ - empty squares in Fig.4) or without them (case $b$-empty circles in Fig.4) for POMME analyses, and all SA terms for CHAOS analysis (case $c$-full squares in Fig.4). Both analyses of POMME models (cases $a$ and $b$ ) were extended up to $N=10$, but no significant change appears with lower and higher degrees (verified for $N=8$ and 16); the analysis of CHAOS models was limited to $N=14$. Also in all these cases we find an exponential growth of the corresponding differences, in particular with characteristic times $\tau=5.2 \pm 0.1$ (case $a$ ), $4.9 \pm 0.1$ (case $b$ ) and 5.1 \pm 0.1 (case $c$ ) years, which are all in agreement with the result from the IGRF analyses (Fig.2a). We notice that when we use POMME 3.0 without its SA terms (case $b$ in Fig.4), we find a comparable characteristic time but the initial error $\varepsilon_{0}$ is almost doubled with respect to the case $a$ with all SA terms. Apparently, this result indicates that any effort to improve the predictive SV with degree higher than $N=8$ is worth doing, although the intrinsic chaotic nature of the SV field does not allow to maintain the same accuracy further into the future. About this argument some other discussions have been recently posed by Silva et al. (2010).

Two important questions arise: i) Was this exponential divergence (possibly implying ergodicity and chaos) between predicted and actual geomagnetic field values already present in an epoch older than 1965 ? ii) How can present conclusions about this predictability limitation to a few years be drawn only from instantaneous variation estimates? That is, could it be possible that some, say, decadal average would give some kind of better predictability? Perhaps short term fluctuations cannot be predicted accurately, but maybe there is a general long-term regularity (periodicity?) so 
that the system is not ergodic on longer time scales. To answer these questions we now consider a longer-term model, i.e. GUFM1 (Jackson et al., 2000): this model is based on a SHA of all available historical ground and marine data from 1590 to 1990.

Figure 5 shows the same kind of analysis applied this time to the GUFM1 model for the periods 1600-1610 and 1900-1910, with the main difference that now we consider decadal SV averages. In both cases we first estimated the SV coefficients from the field coefficients in the 10 years prior each considered epochs, i.e. 1590-1600 and 1890-1900, respectively. Then, on the basis of this averaged SV, we produced the prediction field values for subsequent 10 years and compared them with the real GUFM1 field values for the same period of time. Also in these cases clear exponential growths are evident ( $\tau=7.3$ and 9.7 with the same theoretical uncertainty of \pm 0.1 years).

Taking into account all cases analysed so far, i.e. a total of 20 cases, we can find an overall unweighted mean value $\left\langle\tau>_{\text {all }}\right.$ of 6 years with an uncertainty of around 3 years; also the median of 5.7 years confirms the robustness of this estimation.

The divergence of the error in all analysed cases is evidence for a chaotic geomagnetic field as deduced from a time domain analysis. Within the uncertainty, the mean value $<\tau>$ (overall mean value, but also both mean values from IGRF and WMM analyses) agrees very well with the characteristic time $\langle T>=1 / K \approx 6$ years after which no reliable prediction can be made, as found from the nonlinear analyses of the geomagnetic field performed by De Santis et al. (2002) in the phase space. While the slightly larger $\tau$ values of WMM and GUFM1 analyses could be ascribed to statistical fluctuations around the theoretical value (even if, perhaps, the largest value for GUFM1 analysis at 1900 could be due to some inability of this model to represent the real field at that epoch), their larger uncertainty, associated to the mean values of the characteristic time, is probably due to a different quality of the analysed models over the considered time.

The close agreement between the results from the two separate and independent analyses, i.e. $<\tau>$ deduced from the time error analysis and $\langle T\rangle$ from the NCA in the phase space, can be 
considered an empirical proof of the geomagnetic field ergodicity. From the analyses with GUFM1, it is possible to infer that this property can be extended from 1600 to the present.

\section{Conclusions}

The exponential temporal divergence of the errors between several couples of predictive and definitive global geomagnetic models agrees very well with the results obtained from a previous NCA (e.g. Barraclough and De Santis, 1997; De Santis et al., 2002), thus confirming the present state of a chaotic geomagnetic field with no reliable prediction after around 6 years. The empirical evidence for the ergodicity of the geomagnetic field given by the present work indicates a strong spatio-temporal coupling among the unknown components of the dynamical system behind the geomagnetic field generation, as it was already found independently by analysing the spatial and temporal spectra of global models and geomagnetic observatory time series, respectively (De Santis et al., 2003). The disclosed ergodicity implies that we can simplify many geomagnetic field analyses for estimating some ergodic measures, such as the information content or various kinds of entropies. For instance, instead of constructing the dynamical orbit in the phase space and estimating some kinds of entropy (e.g. Shannon and Kolmogorov Entropies) from successive time delays, we can resort to time averages of the quantity, as proposed by Baranger et al. (2002) in another context. An application of this approach in geomagnetism has been recently shown by De Santis et al. (2004, 2009), De Santis (2007, 2008) and De Santis and Qamili (2010) whose results provide new insights of the present geomagnetic field and its possible evolution into the future.

In addition, three practical considerations can be made. The first concerns with the question that some efforts to improve the representation of the SV (and SA), for instance increasing the maximum index $N$ of their SH expansions, are worth doing (see also Silva et al., 2010), although the intrinsic chaotic nature of the field will not allow the models to maintain the same accuracy for some years into the future. The second consideration regards the geodynamo simulations: it would be really interesting to study the effect of imposing the presence of ergodicity in some geodynamo 
simulations, especially if one of the objectives is to make predictions of the geomagnetic field for the close future. The third consideration concerns with the times when the geomagnetic field models appear more or less chaotic (less or more predictable) than in other epochs: our opinion is that the way of proceed in comparing provisional and definitive global models could be useful to detect and, possibly, confirm the presence of geomagnetic jerks (e.g. Mandea et al., 2010), when we expect the geomagnetic field is less predictable than usual, so more chaotic, i.e. with smaller characteristic time $\tau$.

More studies are needed to verify the important property of ergodicity of the present geomagnetic field also prior to 1600 , and to explore the three additional considerations about global models with more detailed SV and SA terms, ergodic geodynamos and jerks detection.

\section{Acknowledgements}

We would like to thank Stefan Maus and his co-authors (2008) because their paper inspired ours. We also appreciate very much all efforts of those who acquire magnetic data and/or determine geomagnetic models: without their work we could not do anything. Mioara Mandea and an unknown referee helped us with their comments to improve the paper significantly. Gauthier Hulot pointed out some apparent incongruences in a preliminary version of the paper. This work has been made in the frame of the Project REM (“Reversing Earth Magnetism?") funded by the PNRA ("National Program of Research in Antarctica").

\section{References}

Anosov, D.V., 2001. Ergodic theory, in Encyclopaedia of Mathematics (Ed.s Hazewinkel and Michiel). Springer, ISBN 978-1556080104.

Anderson, J.G., Brune, J.N., 1999. Probabilistic hazard analysis without the ergodic assumption. Seism. Res. Lett 70, 19-23. 
Baranger, M., Latora, V., Rapisarda, A., 2002. Time evolution of thermodynamic entropy for conservative and dissipative chaotic maps. Chaos Solitons \& Fractals 13, 471-478.

Barraclough, D.R., De Santis, A., 1997. A fractal interpretation of the topography of the scalar geomagnetic scalar potential at the core-mantle boundary. Pure Appl. Geophys. 149, 747-760.

Buonomano, V., Bartmann, F., 1986. Testing ergodic assumption in the low-intensity interferences experiments. Il Nuovo Cimento 95B No.2, 99-108.

Chen, Y-H., Tsai, C-C. P., 2002. A new method of the attenuation relationship with variance components. Bull. Seism. Soc. Am. 92 No.5, 1984-1991.

Cross, M.C., Hohenberg, P.C., 1994. Spatiotemporal chaos. Science 263, 1569.

De Santis, A., 2007. How persistent is the present trend of the geomagnetic field to decay and, possibly, to reverse?. Phys Earth Planet In. 162, 217-226.

De Santis, A., 2008. Erratum to "How persistent is the present trend of the geomagnetic field to decay and, possibly, to reverse?". Phys Earth Planet In. 170, 149.

De Santis, A., Barraclough, D.R., Tozzi, R., 2002. Nonlinear variability of the Recent Geomagnetic Field. Fractals 10, 297-303.

De Santis, A., Barraclough, D.R., Tozzi, R., 2003. Spatial and temporal spectra of the geomagnetic field and their scaling properties. Phys Earth Planet In. 135, 125-134.

De Santis, A., Qamili, E., 2010. Shannon information of the geomagnetic field for the past 7000 years. Nonlinear Proc Geoph. 17, 77-84.

De Santis, A., Cianchini, G., Qamili, E., Frepoli, A., 2010. The 2009 L'Aquila (Central Italy) seismic sequence as a chaotic process. Tectonophysics $496,44-52$.

Eckmann, J.P., Ruelle, D., 1985. Ergodic Theory of chaos and strange attractors. Rev. Mod. Phys. 57 No.3 Part I, 617-656.

Egolf, D., 2000. Equilibrium Regained: From Nonequilibrium Chaos to Statistical Mechanics. Science 287, 101-104. 
Finlay, C.C., Maus, S., Beggan, C.D., Hamoudi, M., Lowes, F.J., Olsen N., Thebault, E., 2010. Evaluation of candidate geomagnetic field models for IGRF-11. Earth Planets Space 62787 804.

Hongre, L., Sailhac, P., Alexandrescu, M., Dubois, J., 1999. Non linear and multifractal approaches of the geomagnetic field. Phys. Earth Plan. Int. 110, 157-190.

Jackson, A., Jonkers, A.R.T., Walker, M.R., 2000. Four centuries of geomagnetic secular variation from historical records. Phil. Trans. R. Soc. Lond. A 358, 957-990.

Kantz, H., Schreiber, T., 1997. Nonlinear time series analysis. Cambridge Univ. Press.

Mandea, M., Holme, R., Pais, A., Pinheiro, K., Jackson, A., Verbanac, G., 2010. Geomagnetic Jerks: Rapid Core Field Variations and Core Dynamics. Space Sci. Rev., 155, 147-175.

Maus, S., Macmillan, S., Chernova, T., Choi, S., Dater, D., Golovkov, V., Lesur, V., Lowes, F., Luhr, H., Mai, W., McLean, S., Olsen, N., Rother, M., Sabaka, T., Thomson, A., Zvereva, T., 2005. The 10th-Generation International Geomagnetic Reference Field. Geophys. J. Int. 161, $561-565$.

Maus, S., Rother, M., Stolle, C., Mai, W., Choi, S., Lühr, H., Cooke, D., Roth, C., 2006. Third generation of the Potsdam Magnetic Model of the Earth (POMME). Geochem. Geophys. Geosyst. 7, Q07008, doi:10.1029/2006GC001269.

Maus, S., Silva, L., Hulot, G., 2008. Can core-surface flow models be used to improve the forecast of the Earth's main magnetic field? J. Geophys. Res. 113, B08102, doi:10.1029/ 2007JB005199.

Maus, S., Manoj, C., Rauberg, J., Michaelis, I., Lühr, H., 2010. NOAA/NGDC candidate models for the 11th generation International Geomagnetic Reference Field and the concurrent release of the 6th generation POMME magnetic model. Earth Planets Space 62, 729-735.

McLean, S., Macmillan, S., Maus, S., Lesur, V., Thomson, A., Dater, D., 2004. The US/UK World Magnetic Model for 2005-2010. NOAA Tech. Rep. NESDID/NGDC-1. 
Olsen, N., Luhr, H., Sabaka, T.J., Mandea, M., 2006. CHAOS - A model of Earth's magnetic field derived from CHAMP, Oersted, and SAC-C magnetic satellite data. Geophys. J. Int. 166, 6775.

Olsen, N., Mandea, M., Sabaka, T.J., Tøffner-Clausen, L., 2009. CHAOS-2 - A geomagnetic field model derived from one decade of continuous satellite data. Geophys. J. Int. 179, 1477-1487.

Paine, A.D.M., 1985. Ergodic reasoning in geomorphology: time for a review of the term? Progress in Phys. Geography vol.9 no.1, 1-15.

Sabaka, T.J., Olsen, N., Purucker, M.E., 2004. Extending comprehensive models of the Earth's magnetic field with Ørsted and CHAMP data. Geophys. J. Int. 159, 521- 547.

Schuster, H.G., 1995, Deterministic Chaos: an introduction (3rd ed.). Wiley-VCH, Weinheim, pp. 291.

Silva, L., Maus, S., Hulot, G., Thebault, E., 2010. On the possibility of extending the IGRF predictive secular variation model to a higher SH degree. Earth Planets Space 62, 815-820.

Sugihara, G., May, R.M., 1990. Nonlinear forecasting as a way of distinguish chaos from measurement error in time series. Nature 344, 734-741.

Takens, F., 1981. Detecting strange attractors in turbulence. In: Rand, D.A., Young, L.S., (Editors), Lecture notes in mathematics, 898, Springer, Berlin, pp. 366.

Wales, D.J., 1991. Calculating the rate of loss information from chaotic time series by forecasting. Nature $350,485-488$.

Walters, P., 1982. An introduction to ergodic theory. Springer, New York.

Woo, G., Wang, G-Q., Tang, G., 2006. Insights from Parkfield array data for probabilistic risk modelling. Proceed. $8^{\text {th }}$ US National Conference on Earthquake Engineering, San Francisco, Ca, USA, Paper No.219, pp.8. 
Figure 1. Errors for eight temporal segments numbered in progressive order, estimated from the differences between predicted (IGRF and WMM models) and definitive global geomagnetic models in the period 1965-2010, (adapted from Fig.1 of Maus et al., 2008). For the used definitive models see the text for the details.

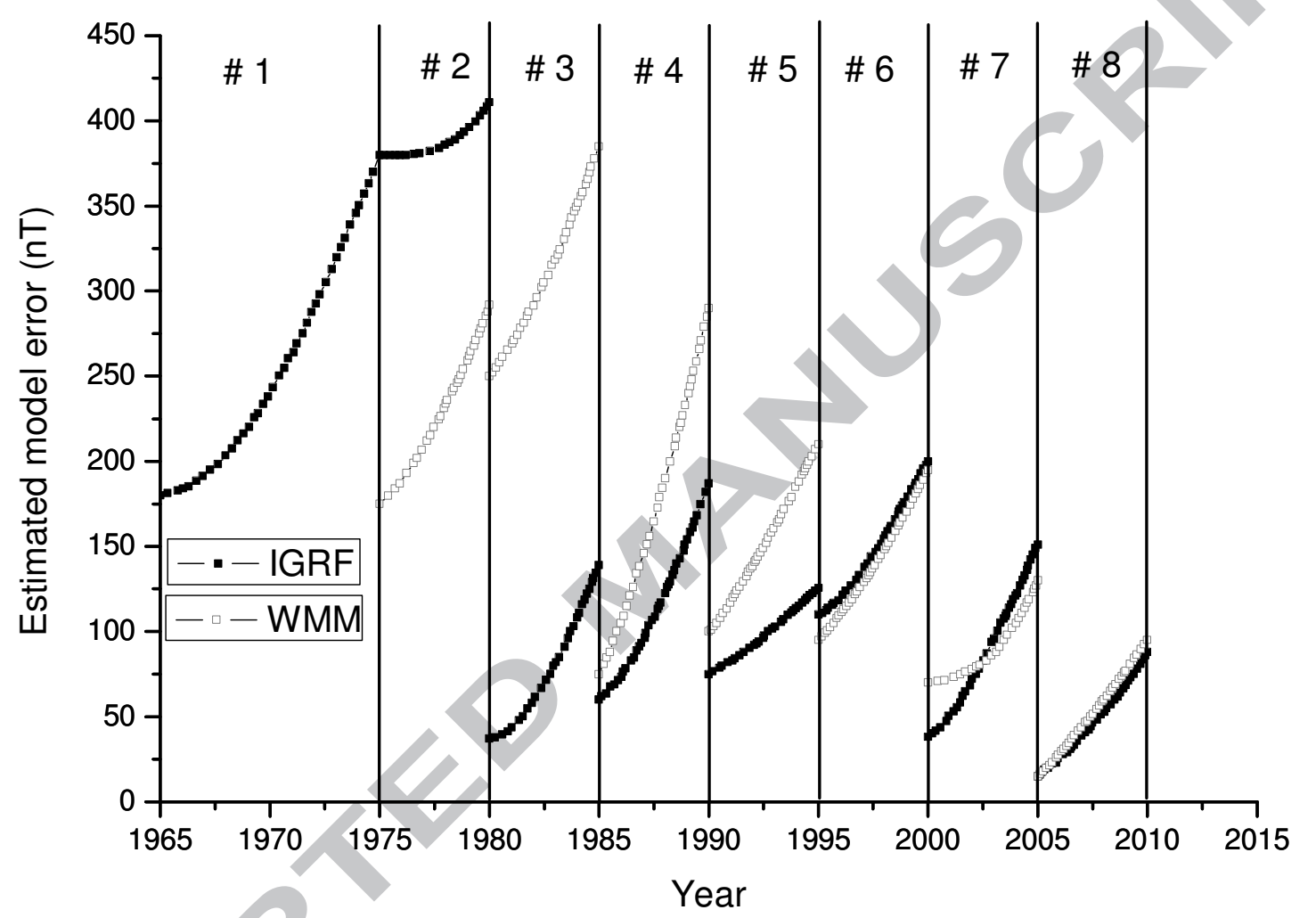


Figure 2. Errors for a) IGRF and b) WMM models, as Fig.1 but imposing the same initial time for a better visual convenience and comparison. All segments (numbered according to the same order of Fig.1) show an exponential growth with characteristic time $\tau$ : in particular, $<\tau>=5.2 \pm 2.3$ (standard deviation) years (median of 5.4 years) for the IGRF model and $<\tau>=7.9 \pm 3.5$ years (median of 7.6 years) for WMM model. Within the estimated uncertainty, these values confirm the characteristic time $T$ as found from the geomagnetic field chaotic analysis made in the phase space by De Santis et al. (2002) and, thus, it empirically proves the ergodicity of the recent geomagnetic field.

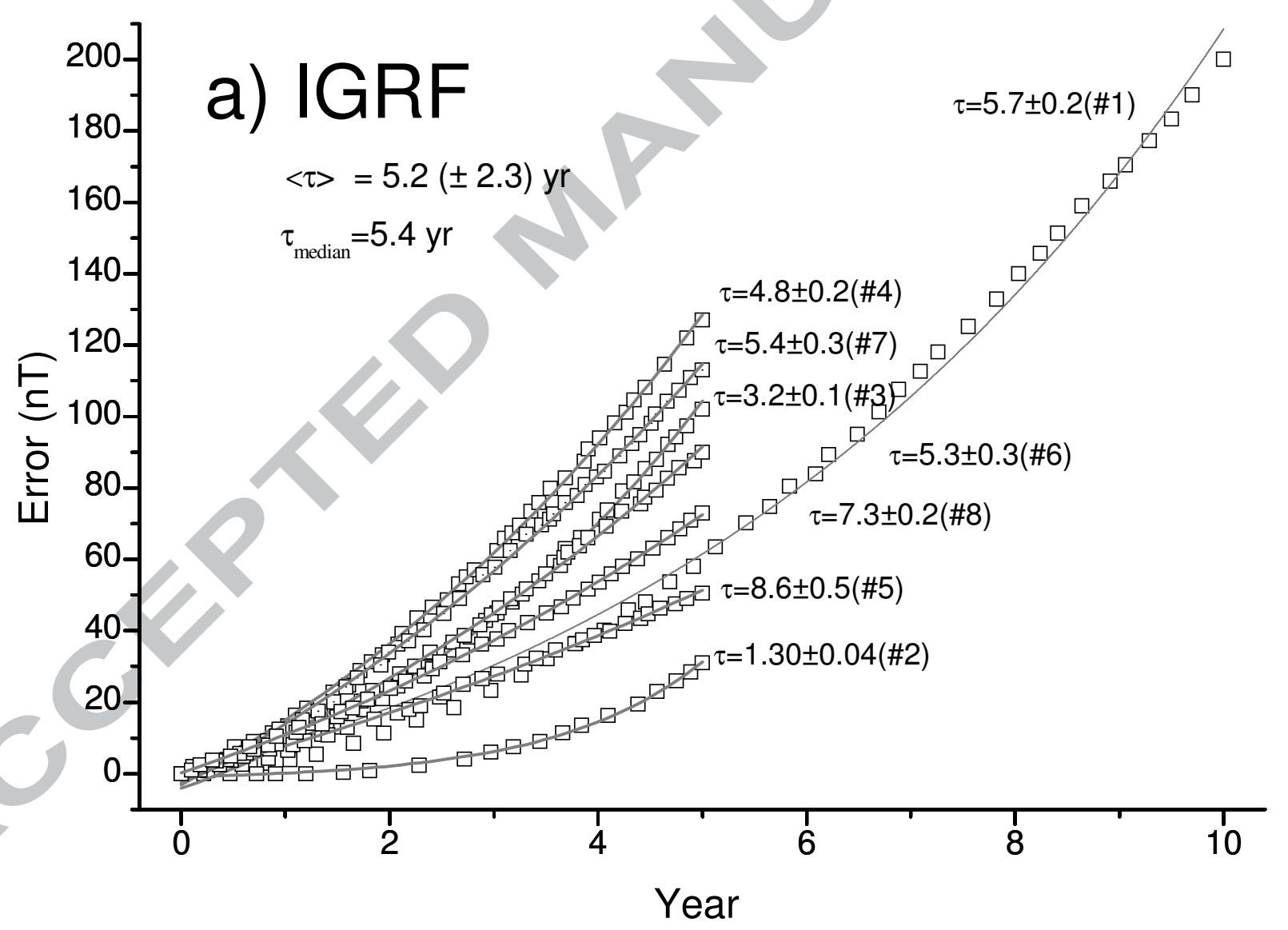




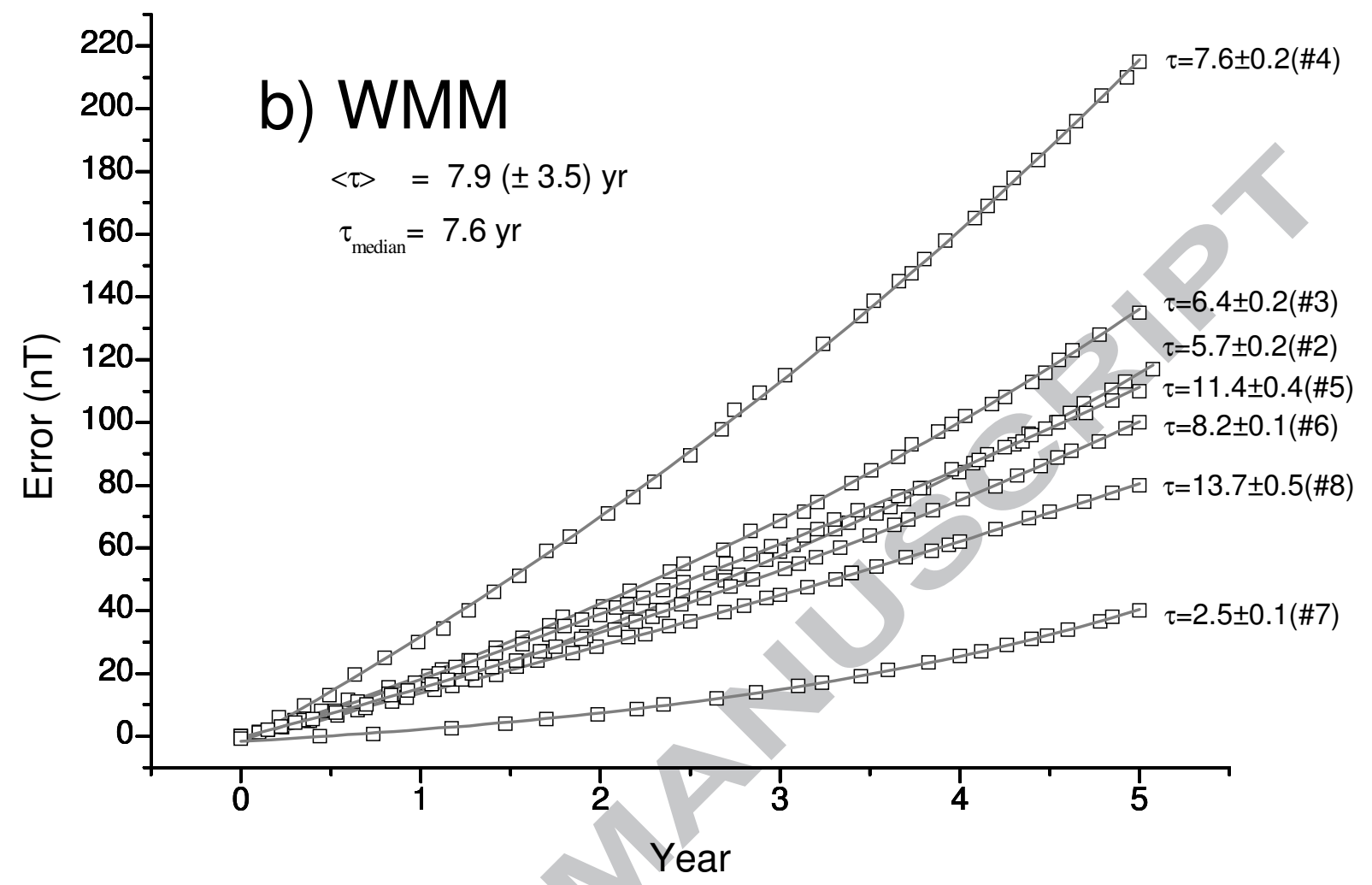


Figure 3. Misfit between CM4 and POMME 3.0 (backward extrapolated from 2002 to 1990) that shows an exponential decay with characteristic time $\tau=5.4 \pm 0.5$ years.

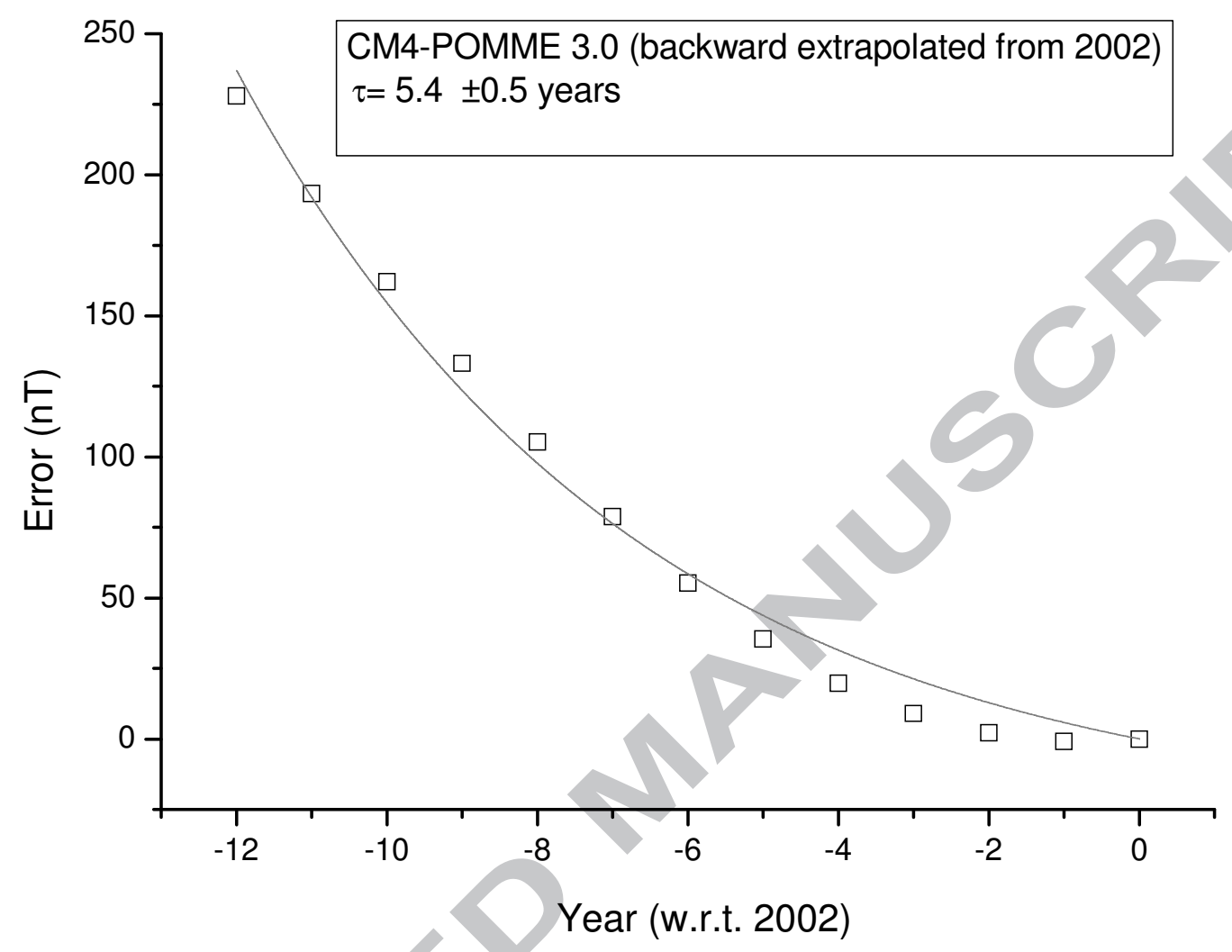


Figure 4. Misfits between POMME 3.0 and POMME 6.0 $(N=10)$ over the period 2006-2010 for both models with MF, SV and SA included (empty squares; case $a$ ) and without for POMME 3.0 (empty circles; case $b$ ). Both curves follow an exponential growth with characteristic time $\tau$ of around 5 years. With $N=8$ and $16, \tau$ does not change significantly within the estimated uncertainty. The case of POMME 3.0 without SA presents a lower quality (higher initial error $\varepsilon_{0}$ ). Case $c$ (full squares) concerns with the analysis of CHAOS-1 and CHAOS-2 differences. Also here we find a characteristic time $\tau$ of around 5 years.

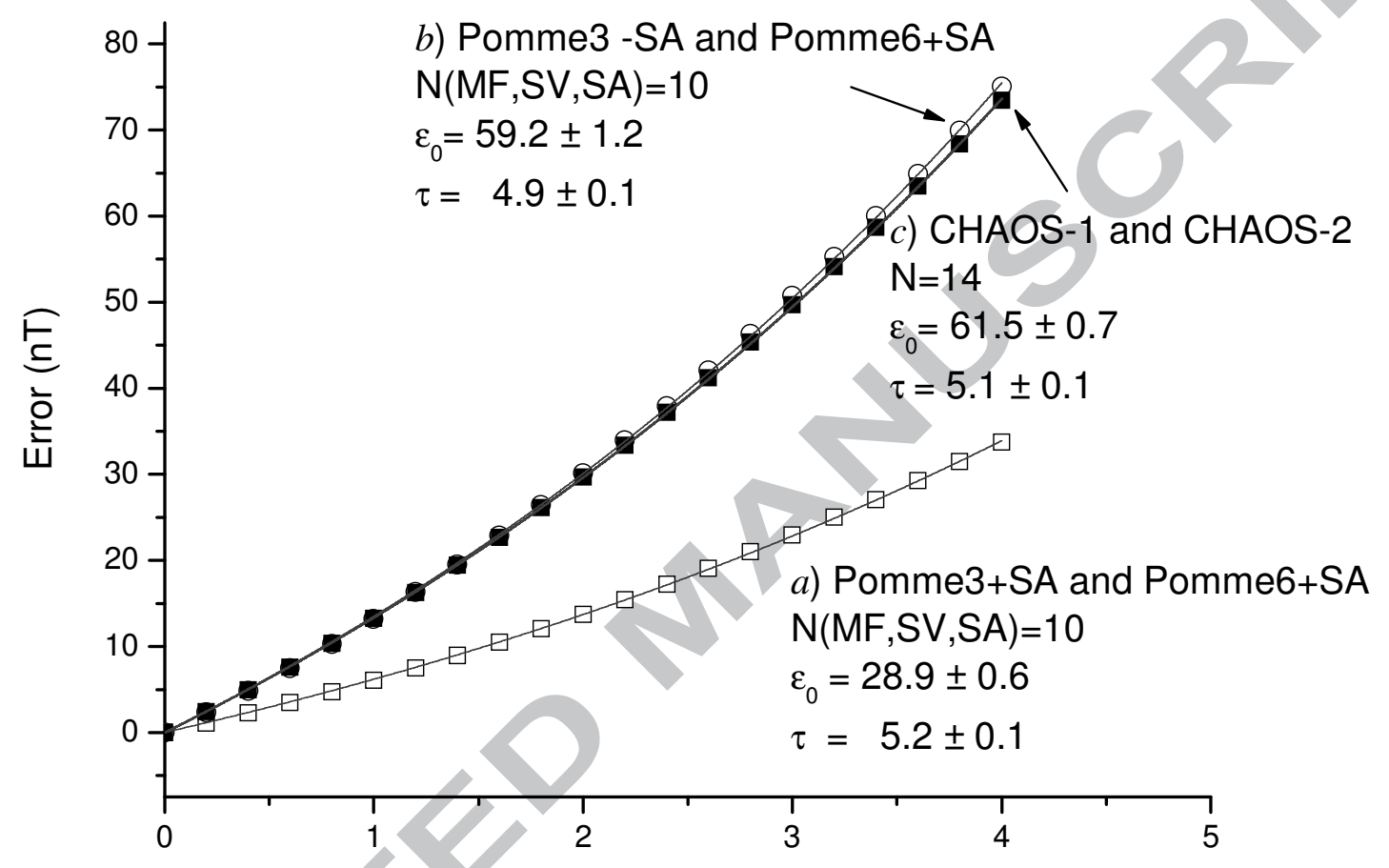

Year from 2006 
Figure 5. Misfits from GUFM1 model at epochs 1600-1610 (a) and 1900-1910 (b). Both show an exponential growth with characteristic time $\tau=7.3( \pm 0.1)$ and $9.7( \pm 0.1)$ years, respectively. See text for more details.

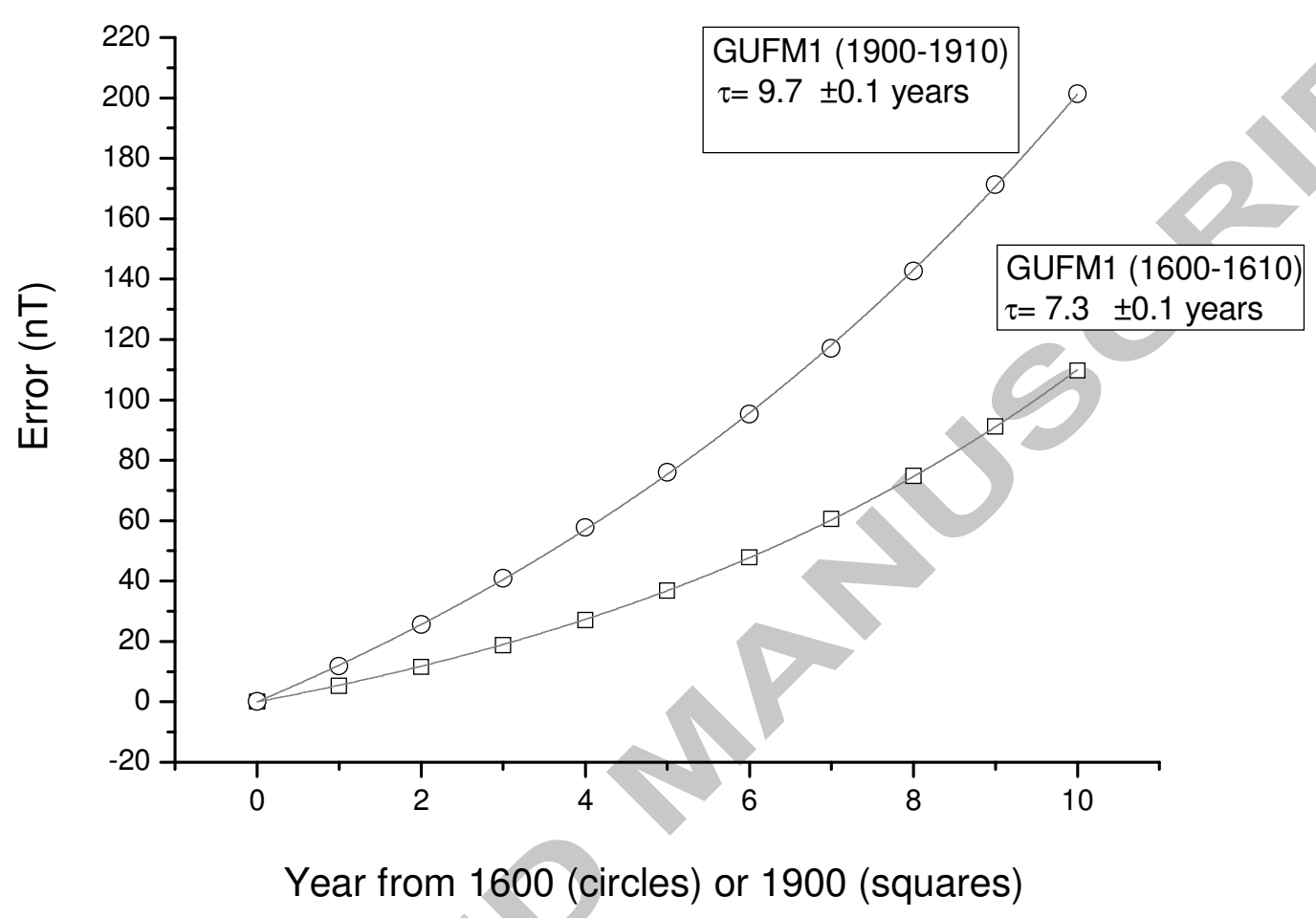




\section{Highlights}

$>$ Ergodic property of the recent geomagnetic field is investigated. > Time deviations between predictive and definitive global models are analyzed. > The results confirmed a chaotic Main Field and highlighted its ergodic character. > The results would allow the estimation of entropic field quantities by time averages. > Potential use of the method for improving geodynamo simulations and jerks detection. 